\title{
New Clues in the Malignant Progression of Glioblastoma: Can the Thioredoxin System Play a Role?
}

\author{
Fatih ERDI ${ }^{1}$, Bulent KAYA ${ }^{1}$, Hasan ESEN² , Yasar KARATAS ${ }^{1}$, Siddika FINDIK ${ }^{2}$, Fatih KESKIN¹, \\ Bahadir FEYZIOGLU ${ }^{3}$, Erdal KALKAN ${ }^{1}$ \\ ${ }^{1}$ Necmettin Erbakan University, Meram Faculty of Medicine, Department of Neurosurgery, Konya, Turkey \\ ${ }^{2}$ Necmettin Erbakan University, Meram Faculty of Medicine, Department of Pathology, Konya, Turkey \\ ${ }^{3}$ Necmettin Erbakan University, Meram Faculty of Medicine, Department of Clinical Microbiology, Konya, Turkey \\ This study was presented at the $30^{\text {th }}$ National Meeting of the Turkish Neurosurgical Society in 8-12 April 2016, Antalya, Turkey
}

\section{ABSTRACT}

AIM: To evaluate and compare the expression of thioredoxin reductase 1 (TrxR1) in primary and secondary glioblastoma samples.

MATERIAL and METHODS: Surgically resected human glioblastoma samples from 40 patients who underwent surgery at our institution were extracted from their histopathological specimens and divided into three groups. Ten histopathologically regular cerebral tissue samples, acquired from the non-neoplastic portion of the specimens, were assigned as the control group. Twenty specimens that included tumoral tissue from each type of glioblastoma (WHO grade IV, primary and secondary) were assigned as the primary and secondary glioblastoma groups. TrxR1 expression was analyzed by using both quantitative reverse transcription polymerase chain reaction (qRT-PCR) and immunohistochemistry. Isocitrate dehydrogenase 1 (IDH1) mutation was analyzed by immunohistochemistry. Ki-67 proliferative index and apoptosis were also analyzed by immunohistochemistry. The differences between the groups were statistically compared and the correlation between these parameters was analyzed.

RESULTS: The expressions of TrxR1 and Ki-67 values were significantly higher in primary glioblastoma. IDH1 mutation was significantly higher in secondary glioblastoma. TrxR1 expression was found to be highly correlated with the Ki-67 index. The apoptotic index was similar between primary and secondary glioblastoma.

CONCLUSION: This study showed a high TrxR1 expression in primary glioblastoma which could indicate a role of the Trx system in promoting the malignant progression by some complex processes.

KEYWORDS: Thioredoxin reductase 1, Isocitrate dehydrogenase 1, Glioblastoma, Primary, Secondary, Mutation

\section{INTRODUCTION}

$\checkmark$ lioblastoma has two subtypes based on clinical and genetic findings including primary glioblastoma, which arises de novo, and secondary glioblastoma, which progress from low grade gliomas to glioblastoma (7). Primary glioblastomas develop rapidly de novo without clinical or histological evidence of a less malignant precursor lesion, manifest especially in elderly patients. Secondary glioblastomas progress from a less malignant precursor lesion like as low-grade diffuse astrocytoma or anaplastic astrocytoma. Secondary glioblastomas are usually seen in younger patients and have less necrosis with significantly better prognosis (16). Determinative genetic signposts of secondary glioblastoma are isocitrate dehydrogenase 1 (IDH1) mutations, which are absent in primary glioblastomas $(10,16)$.

Thioredoxin (Trx) and thioredoxin reductase (TrxR) are redoxactive proteins that participate in various cellular events such as promotion of growth, apoptosis, and cellular defense 
mechanisms (19). The relevant literature indicates that the level of TrxR is increased in glioblastoma patients (12). A significant increase of thioredoxin reductase 1 (TrxR1) in astrocytomas according to their ascending grades has been shown previously (5).

In the present study, we examine and compare TrxR1 expression, IDH1 mutation, $\mathrm{Ki}-67$ and apoptotic index patterns of surgically resected primary and secondary glioblastoma tissues by using quantitative reverse transcription polymerase chain reaction (qRT-PCR) and immunohistochemistry.

\section{MATERIAL and METHODS}

\section{Patient Group}

Surgically resected human glioblastoma samples from 40 patients who underwent surgery at our institution from 2006 to 2013 were selected for this study. Fixed specimens in $4 \%$ phosphate-buffered formaldehyde were extracted into paraffin-embedded blocks. Two experienced neuropathologists conducted the histopathological investigations such as typing and grading of the hematoxylin and eosin (H\&E)-stained slides according to the World Health Organization (WHO) criteria (13). Ten histopathologically regular cerebral tissue samples acquired from the non-neoplastic portion of the specimens were assigned as the control group. Twenty specimens, which included tumoral tissue from each type of glioblastoma (WHO grade IV, primary and secondary), were assigned as the primary and secondary glioblastoma groups. The patient groups had not received chemotherapy or radiotherapy before surgery. Informed consent from patients or their primary relatives and ethical approval from local ethics committee of our institution were obtained.

\section{RNA Isolation and qRT-PCRs}

Ribonucleic acid (RNA) isolation and qRT-PRC evaluation were done in a standard fashion similar to the literature (5). Briefly, total RNA was isolated from formalin-fixed, paraffinembedded (FFPE) tissues using the High Pure FFPET RNA Isolation Kit (Roche Applied Science, cat. 6868517), according to the manufacturer's instructions. The Transcriptor First Strand c-DNA Synthesis Kit (Roche Applied Science) was used to generate c-DNA from RNA, according to the manufacturer's instructions; $10 \mu \mathrm{L}$ total RNA was used for amplification. Amplification reactions were set up in a reaction volume of $20 \mu \mathrm{L}$ using the LightCycler 480 PCR Master Mix (Roche Applied Science, cat. 04707494001). PCR primaries and TaqMan probes (Roche Applied Science, cat. 05532957001) were synthesized and preoptimized. qRTPCR was performed using PCR primaries (human TrxR1 forward,5'-CCGCAACAGCCAAAATGGTGA-3', and reverse, 5'-AGCATGATT AGGCAAACTCCGTAA-3'; and human GAPDH forward, 5'-GACTCATGACCACAGTCCATGC-3', and reverse 5'- AGAGGCAGGGATGATGTTCTG-3' and TaqMan probes in the LightCycler 480 II system (Roche Applied Science). Amplification was performed for 40 cycles at $95^{\circ} \mathrm{C}$ for 10 seconds, followed by $60^{\circ} \mathrm{C}$ for 30 seconds, and $72^{\circ} \mathrm{C}$ for 1 second. Quantitation was achieved using the comparative CT method, normalizing against the glyceraldehyde 3-phosphate dehydrogenase (GAPDH) gene. The expression levels in tumor tissues relative to the non-tumor controls were assessed using the $2^{-\Delta \Delta C t}$ method. Briefly, the threshold cycle (Ct) of fluorescence for each sample was determined. $\Delta \mathrm{Ct}$ indicates the difference in expression levels between TrxR1 and GAPDH $\left(\Delta \mathrm{Ct}=\mathrm{Ct}_{\mathrm{TrRR} 1}-\mathrm{Ct}_{\mathrm{GAPDH}}\right)$, and $\Delta \Delta \mathrm{Ct}$ indicates the difference in the $\Delta \mathrm{Ct}$ value between the tumor tissue and the control $(\Delta \Delta \mathrm{Ct}=$ $\left.\Delta \mathrm{Ct}_{\text {tumor }}-\Delta \mathrm{Ct}_{\text {control }}\right)$. The $2^{-\Delta \Delta \mathrm{Ct}}$ value (fold-value) was calculated.

\section{Immunohistochemistry}

Immunohistochemical evaluation was done in a standard fashion similar to the literature (5). Previously paraffinembedded specimens were sectioned (4 $\mu \mathrm{m}$ thickness) and mounted onto positively charged slides (Objektträger, Germany). Immunohistochemistry was then performed using an automated avidin biotin system (Ventana Benchmark XT, Ventana Medical Systems, Tucson, AZ, USA) employing specific commercially available antibodies.

The Ki-67 index was evaluated by using a monoclonal antibody (Thermo Fisher Scientific Inc., MA, USA), TrxR1 expression was evaluated by using anti-TrxR1 antibody (Anti-TXNRD1 Polyclonal Antibody, Bioss, MA, USA). Immunohistochemistry for IDH1 mutation was evaluated by commercially available monoclonal antibody (MABC171, AntiIsocitrate Dehydrogenase 1 (IDH1)-R132H Antibody, clone HMab-1, Merck Millipore, Darmstadt, Germany). Cell nuclei with any brown coloration were considered Ki-67 positive, regardless of the intensity or pattern of staining. Ki-67 positive nuclei were counted in ten areas selected at random, using a $x 40$ objective. The procedure was repeated twice for each specimen and the counts averaged. Ki-67 positivity was calculated as the number of immunoreactive nuclei per total number of cells.

For TrxR1, the cytoplasmic immunoreactivity patterns were categorized as negative (0), weak (1), moderate (2), or intense (3) as reported previously (8).

For the evaluation of IDH1 mutation, combined cytoplasmic and nuclear staining was interpreted as immunopositive. The results were accepted as negative if no tumor cell was immunopositive and positive if a focal or diffuse immunopositivity was detected (1).

\section{Immunostaining for Apoptosis}

Immunohistochemical evaluation was done in a standard fashion similar to the literature (5). The ApopTag Peroxidase In Situ Apoptosis Detection Kit (Chemicon International, Apoptosis Detection Kit, S7101, CA, USA) was used to demonstrate apoptosis in a cross-section of non-necrotic tumoral tissues. Paraffin-embedded samples were deparaffinized and rehydrated in decreasing concentrations of alcohol. Samples were first treated with proteinase $\mathrm{K}(20 \mathrm{mg} / \mathrm{mL})$ for 15 minutes at room temperature to increase their permeability. After blocking of endogenous peroxidase activity by incubation with $3 \%$ $\mathrm{H}_{2} \mathrm{O}_{2}$ for 5 minutes, $75 \mathrm{~mL}$ equilibration buffer was applied to the sections. The samples were then treated with terminal deoxynucleotidyl transferase (TdT enzyme) in a humidified chamber for 1 hour at room temperature. TdT catalyzes the 
polymerization of nucleotides to free 3'-OH DNA ends in a template-independent manner and is used to label DNA strand breaks. Sections were then incubated with an anti-digoxigenin peroxidase conjugate for 30 minutes. To develop color, a peroxidase substrate was applied for 3-6 minutes. Samples were subsequently counter-stained with hematoxylin and mounted. Between steps, the slides were washed in phosphate-buffered saline (PBS). Nuclear staining was observed in the cell nuclei of the tumor sections. The apoptotic index was estimated as the number of immunoreactive nuclei per total number of cells and expressed as a percentage.

\section{Statistical Analysis}

SPSS for Windows 18.0 (SPSS, Inc., Chicago, IL, USA) was used for statistical analyses. Obtained data was expressed as median, minimum-maximum or mean \pm standard deviation (SD). The Kruskal-Wallis test was used. Pairwise comparisons were analyzed by the Mann-Whitney test with Bonferroni adjustment. Correlation analysis was done with Spearman's rank correlation coefficients. $\mathrm{p}<0.05$ was considered statistically significant.

\section{RESULTS}

The primary glioblastoma group showed significantly higher TrxR1 expression in both qRT-PCR and immunohistochemical evaluations when compared with the control and secondary glioblastoma groups $(p<0.05)$. The results of qRT-PCR experiments are summarized in Table $I$ and the results of immunostaining can be seen in Figure 1A-O.

The IDH 1 mutation was significantly higher in secondary glioblastoma $(p<0.05)$. Seven samples from secondary glioblastoma and one sample from primary glioblastoma showed IDH1 mutation. Other samples showed negative staining for IDH1 mutation and were referred to as wild type IDH1.

The Ki-67 index was significantly high in primary glioblastoma $(p<0.05)$. The Ki-67 index and the expression of TrxR1 were found to be highly correlated.

The apoptotic index of primary glioblastoma was significantly higher than in the control group $(p<0.05)$ but the difference between glioblastoma groups was not significant $(p>0.05)$.

The results of immunostaining are summarized in Table II and representative images of each group are presented in Figure $1 \mathrm{~A}-\mathrm{O}$.

\section{DISCUSSION}

Growth signaling independency, insensibility to growth inhibitory signaling, sparing from apoptosis, limitless replication capacity, continuous angiogenesis and tissue invasion capacity are the hallmarks for the development of neoplasia. Although the conceptual importance, type and time trend of interaction were unclear, increasing evidence suggest that Trx signaling involves in a variety of these neoplastic processes (9).

The Trx system harbors ribonucleotide reductase, overexpression of which potentially leads to expanded and imbalanced mutagenic deoxynucleotide pools $(2,9,15)$. It has been suggested that the Trx system may support the progress of a malignant phenotype by influencing major genetic rearrangements, gene amplifications, loss of growth control and resistance to therapy $(2,6)$.

Söderberg et al. indicated an anti-apoptotic role for the $\operatorname{Tr} x$ system through various pathways including a reduction in

Table I: Fold Change in Expression of TrxR1 Gene Relative to the Reference Gene GAPDH in Control and Glioblastoma Groups

\begin{tabular}{|c|c|c|c|c|c|}
\hline Sample & GAPDH $C_{t}$ & $\operatorname{TrxR1} \mathrm{C}_{\mathrm{t}}$ & $\begin{array}{c}\Delta C_{t} \text { (Avg. TrxR1- Avg. } \\
\text { GAPDH) }\end{array}$ & $\begin{array}{c}\Delta \Delta C_{t} \\
\left(\text { Avg. } \Delta \mathrm{Ct}^{-} \text {Avg. }\right. \\
\left.\Delta \mathrm{C}_{\mathrm{t}} \text { control } \pm \mathrm{SD}\right)\end{array}$ & $\begin{array}{c}\text { Normalized TrxR1 mRNA } \\
\text { expression relative to control } \\
2^{\Delta \Delta C t} \pm S D(\min , \text { max) }\end{array}$ \\
\hline Control & 26.87 & 25.76 & -1.11 & 0 & $1(0.87-1.19)$ \\
\hline Secondary glioblastoma & 29.39 & 24.43 & -4.96 & -3.85 & $14.14 \pm 4.04(8.52-21.10)$ \\
\hline Primary glioblastoma & 29.85 & 24.33 & -5.52 & -4.41 & $21.30 \pm 4.4(14.83-31.22)$ \\
\hline
\end{tabular}

Table II: Immunostaining Results

\begin{tabular}{lcccccc}
\hline \multirow{2}{*}{ Immunostaining } & \multicolumn{2}{c}{ Control } & \multicolumn{2}{c}{ Secondary glioblastoma } & \multicolumn{2}{c}{ Primary glioblastoma } \\
\cline { 2 - 7 } & Mean \pm SD & Median (min-max) & Mean \pm SD & Median (min-max) & Mean \pm SD & Median (min-max) \\
\hline Apoptotic index & $0.1 \pm 0.31$ & $0(0-1)$ & $1 \pm 1.17$ & $1(0-4)$ & $1.3 \pm 1.4$ & $1(0-5)$ \\
\hline Ki-67 index & $0.1 \pm 0.31$ & $0(0-1)$ & $17.85 \pm 5.63$ & $17.50(10-30)$ & $27.05 \pm 5.67$ & $25.50(20-38)$ \\
\hline TrxR1 & $0.2 \pm 0.42$ & $0(0-1)$ & $2,30 \pm 0,47$ & $2(2-3)$ & $2.9 \pm 0.3$ & $3(2-3)$ \\
\hline IDH1 Mutation & 0 & $0(0-0)$ & $0.35 \pm 0.48$ & $0(0-1)$ & $0.05 \pm 0.22$ & $0(0-1)$ \\
\hline
\end{tabular}

The values were presented as median and mean $\pm S D$. 


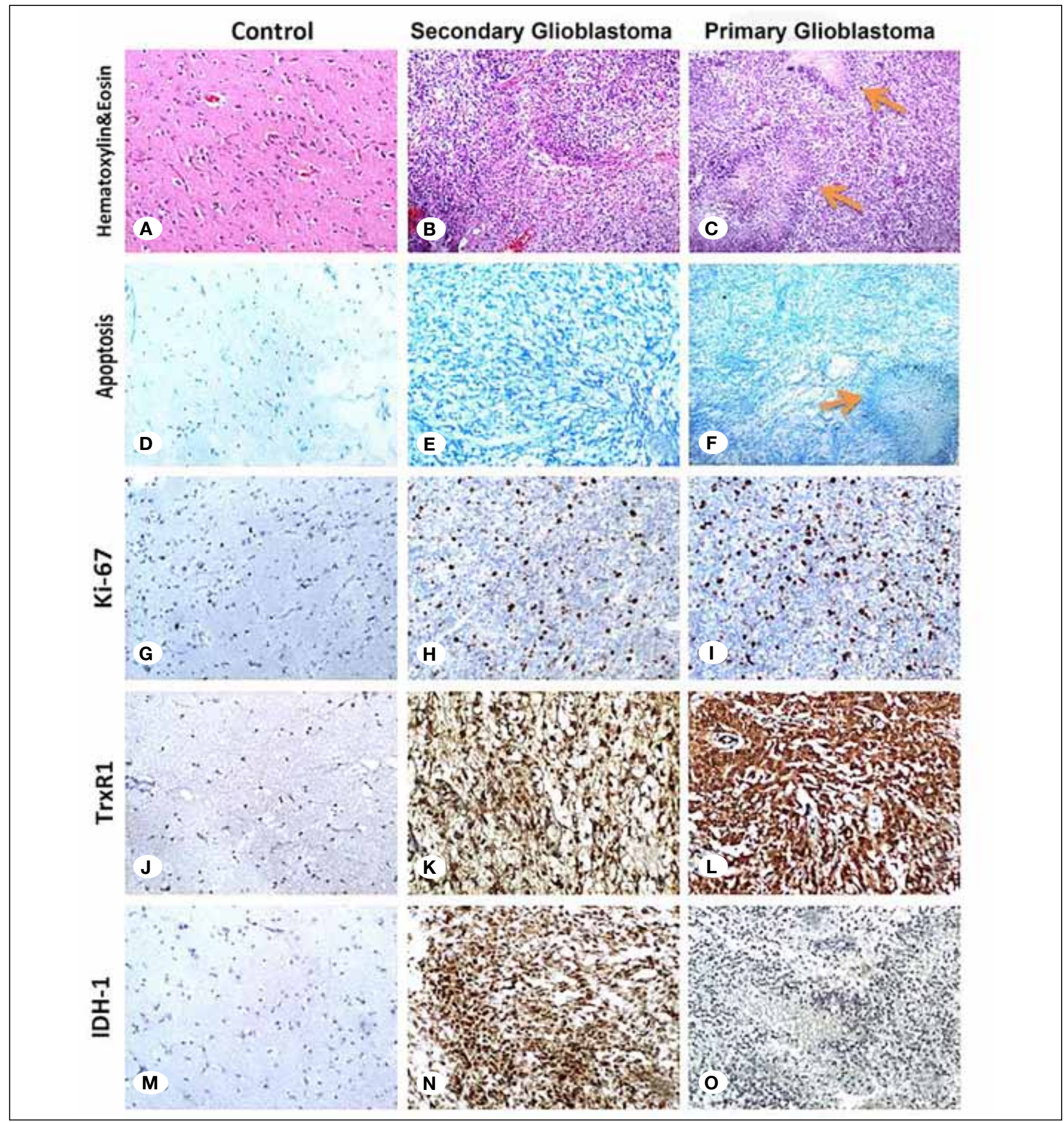

Figure 1: Representative images of control, primary and secondary glioblastoma groups. H\&E staining and immunostaining images for apoptosis, Ki-67,TrxR1 and mutant IDH1. A) Normal brain tissue (H\&E, x100). B) Secondary glioblastoma (H\&E, x40). C) Primary glioblastoma (H\&E, $x 40)$ the arrows indicate palisading necrosis areas. D) Normal brain tissue showed negative staining for apoptosis (TUNNEL, x100). E) Secondary glioblastoma showed negative staining for apoptosis (TUNNEL, x100). F) Primary glioblastoma showed negative staining for apoptosis in non-necrotic area but positive staining in necrotic area (arrow), (TUNNEL, x40). G) Normal brain tissue showed negative Ki-67 staining (x100). H) Secondary glioblastoma showed 10\% Ki-67 positivity (x100). I) Primary glioblastoma showed 25\% Ki-67 positivity (x100). J) Negative staining for TrxR1 in normal brain tissue (x100). K) Moderate staining for TrxR1 in secondary glioblastoma (x100). L) Intense staining for TrxR1 in primary glioblastoma (x100). M) Negative staining for mutant IDH1 in control group (x100). N) Positive staining for mutant IDH1 in secondary glioblastoma (x100). O) Negative staining for mutant IDH1 in primary glioblastoma (x40). 
intracellular hydrogen peroxide via Trx peroxidase, direct inhibition of the apoptosis signal regulating kinase and encouraging cytokine discharge, which ensure the expression of Bcl-2 (19). TrxR1 could play an important role in tumor progression and resistance to chemotherapy through its antiapoptotic functions (11).

The effect of the thioredoxin system in astrocytomas has been previously investigated in several studies. Haapasolo et al. reported more intense Trx and TrxR immunostaining for diffuse astrocytomas when compared with pilocytic astrocytomas (8). Kemerdere et al. reported significantly high TrxR levels in serum and tumoral tissues of glioblastoma patients (12). Recently, Esen et al. showed that TrxR1 was overexpressed in human astrocytomas according to their ascending grades (5).

In this study, we analyzed and compared the level of TrxR1 expression, IDH1 mutation, Ki-67 and apoptotic indices of primary and secondary glioblastoma groups and normal cerebral tissue acquired from non-neoplastic sections of histopathological specimens which served as a control group. This methodology was previously used in other studies $(5,6)$.

Histologically, primary and secondary glioblastomas are usually similar, but they differ in their genetic and epigenetic profiles. Determinative genetic signposts of secondary glioblastoma are IDH1 mutations, which are absent in primary glioblastomas $(10,16)$. IDH1 mutant glioma patients survive longer than wild type patients. According to multivariable analyses, IDH1 mutation can be an independent positive prognostic factor for glioblastomas (17).

We found significantly high IDH1 mutation and significantly low TrxR1 expression in secondary glioblastoma tissues. The $\mathrm{Ki}-67$ index was significantly high in the primary glioblastoma group which demonstrates high proliferative activity. Ki-67 immunostaining is the most commonly used proliferative activity assessment method and is well correlated with the grade and prognosis of human gliomas (18). The apoptotic index of primary glioblastoma was significantly higher than the control group, but the difference between glioblastoma groups was not significant.

In normal mammalian cells, IDH catalyzes the oxidative decarboxylation of isocitrate to a-ketoglutarate and reduces nicotinamide adenine dinucleotide phosphate $(\mathrm{NADP}+)$ to NADPH which also regulates the activity of Trx by TrxR in a substance-dependent manner $(3,14,17)$. In glioma cells, IDH1 mutant enzyme reduces a-ketoglutarate to d-2hydroxyglutarate while converting NADPH to NADP+. In other words, IDH1 mutant enzyme consumes NADPH as it converts a-ketoglutarate to 2-hydroxyglutarate and IDH1 mutant tissues have lower NADPH production, which is a key reducing equivalent supplying the major scavenging systems in a matrix like $\operatorname{Trx}(4)$.

The combination of our findings with the literature indicates high TrxR1 expression in primary IDH1 wild type glioblastoma, which is a more aggressive neoplasm, and lower TrxR1 expression with enhanced IDH mutation in secondary glioblastoma, which is a relatively less aggressive neoplasm $(1,4,5,17)$. As indicated previously, this may be an explanation of increased sensitization to irradiation and chemotherapy and subsequent prolonged survival of IDH1 mutant secondary glioblastoma patients (4).

\section{CONCLUSION}

This study has shown that high TrxR1 expression in primary glioblastoma might refer to a role of the Trx system in supporting astrocytoma progression by some complicated and relatively obscure processes. Further studies are required to explain the exact molecular pathways.

\section{REFERENCES}

1. Agarwal $S$, Sharma MC, Jha P, Pathak P, Suri V, Sarkar C, Chosdol K, Suri A, Kale SS, Mahapatra AK, Jha P: Comparative study of IDH1 mutations in gliomas by immunohistochemistry and DNA sequencing. Neuro Oncol 15(6):718-726, 2013

2. Arnér ESJ, Holmgren $A$ : The thioredoxin system in cancer. Semin Cancer Biol 16(6):420-426, 2006

3. Biaglow JE, Miller RA: The thioredoxin reductase/thioredoxin system: Novel redox targets for cancer therapy. Cancer Biol Ther. 4(1):6-13, 2005

4. Bleeker FE, Atai NA, Lamba S, Jonker A, Rijkeboer D, Bosch KS, Tigchelaar W, Troost D, Vandertop WP, Bardelli A, Van Noorden CJ: The prognostic IDH1(R132) mutation is associated with reduced NADP+-dependent IDH activity in glioblastoma. Acta Neuropathol 119(4):487-494, 2010

5. Esen H, Erdi F, Kaya B, Feyzioglu B, Keskin F, Demir LS: Tissue thioredoxin reductase-1 expression in astrocytomas of different grades. J Neurooncol 121(3):451-458, 2015

6. Esen H, Feyzioglu B, Erdi F, Keskin F, Kaya B, Demir LS: High thioredoxin reductase 1 expression in meningiomas undergoing malignant progression. Brain Tumor Pathol 32(3):195-201, 2015

7. Furuta M, Weil RJ, Vortmeyer AO, Huang S, Lei J, Huang TN, Lee YS, Bhowmick DA, Lubensky IA, Oldfield EH, Zhuang $Z$ : Protein patterns and proteins that identify subtypes of glioblastoma multiforme. Oncogene 23(40):6806-6814, 2004

8. Haapasalo H, Kylaniemi M, Paunul N, Kinnula VL, Soini Y: Expression of antioxidant enzymes in astrocytic brain tumors. Brain Pathol 13(2): 155-164, 2003

9. Hanahan D, Weinberg RA: The hallmarks of cancer. Cell 100(1):57-70, 2009

10. Ishizawa $K$, Hirose $T$, Sugiyama $K$, Kageji T, Nobusawa S, Homma T, Komori T, Sasaki A: Pathologic diversity of glioneuronal tumor with neuropil-like islands: A histological and immunohistochemical study with a special reference to isocitrate dehydrogenase 1 (IDH1) in 5 cases. Clin Neuropathol 31(2):67-76, 2012

11. Iwasawa S, Yamano Y, Takiguchi Y, Tanzawa H, Tatsumi K, Uzawa K: Upregulation of thioredoxin reductase 1 in human oral squamous cell carcinoma. Oncol Rep 25(3):637-644, 2011

12. Kemerdere R, Kacira T, Hanimoglu H, Kucur M, Tanriverdi T, Canbaz B: Tissue and plasma thioredoxin reductase expressions in patients with glioblastoma multiforme. $\mathrm{J}$ Neurol Surg A Cent Eur Neurosurg 74(4):234-238, 2013 
13. Louis DN, Ohgaki H, Wiestler OD, Cavenee WK, Burger PC, Jouvet A, Scheithauer BW, Kleihues P: The 2007 WHO classification of tumours of the central nervous system. Acta Neuropathol 114(2):97-109, 2007

14. Nakamura H: Thioredoxin and its related molecules: Update 2005. Antioxid Redox Signal 7(5-6):823-828, 2005

15. Nordberg J, Arnér ES: Reactive oxygen species, antioxidants, and the mammalian thioredoxin system. Free Radic Biol Med 31(11):1287-312, 2001

16. Ohgaki $H$, Kleihues $P$ : The definition of primary and secondary glioblastoma. Clin Cancer Res 19(4):764-772, 2013
17. Reitman ZJ, Yan H: Isocitrate dehydrogenase 1 and 2 mutations in cancer: Alterations at a crossroads of cellular metabolism. JNCI J Natl Cancer Inst 102(13):932-941, 2010

18. Skjulsvik AJ, Mørk JN, Torp MO, Torp SH: Ki-67/MIB-1 immunostaining in a cohort of human gliomas. Int J Clin Exp Pathol 7(12):8905-8910, 2014

19. Söderberg A, Sahaf B, Rosén A: Thioredoxin reductase, a redox-active selenoprotein, is secreted by normal and neoplastic cells: Presence in human plasma. Cancer Res 60(8):2281-2289, 2000 